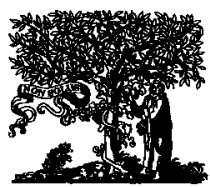

ELSEVIER
International Journal of Pediatric Otorhinolaryngology

32 (Suppl.) (1995) S103-S105

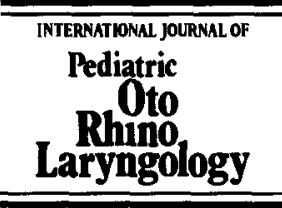

\title{
Wound healing of laryngeal trauma and the development of subglottic stenosis
}

\author{
H.L. Verwoerd-Verhoef*a, J.K. Bean ${ }^{\text {b }, ~ F . C . P . M . ~ A d r i a a n s e n ~}{ }^{\mathrm{c}}$, \\ C.D.A. Verwoerd ${ }^{\mathrm{b}}$ \\ "Department of Otorhinolaryngology, Erasmus University. Rotterdam, The Netherlands \\ ${ }^{\circ}$ Department of Otorhinolaryngology, Erasmus University Rotterdam/Sophia Children's Hospital. \\ Rotterdam, Dr. Molewaterplein 40,3015 GD Rotterdam. The Netherlands \\ 'Dept. of Otorhinolaryngology, Catharina Hospital Eindhoven, Michelangelolaan 2. 5623 EJ Eindhoven, \\ The Netherlands
}

Investigations of wound healing of the cricoid cartilage, its epithelium and subepithelial layer were performed in an experimental model. Each experiment comprised 10 young rabbits at the age of 4 weeks with a standard follow-up period of 20 weeks (to adulthood).

Endolaryngeal lesions could be restricted to the soft tissue lining (epithelium + subepithelial layer), or they could also involve the perichondrium and the inner side of the cartilage. Splits were performed at different sites: anteriorly, bilaterally or antero-posteriorly, along with additional combinations. Assessment was performed by endoscopy and histopathology. The latter comprised $5-\mu \mathrm{m}$ sectioning in the horizontal and sagittal planes. The structure of the larynx of rabbits remarkably corresponds with that of man: the organization of the different cartilaginous parts and their interrelation, the structure of the respiratory epithelium and the subepithelium with vessels, glands, and most important, the elastic system [1].

An endolaryngeal injury with traumatization of the epithelium, the subepithelial layer, and the internal side of the cricoid can lead to major anomalies just 1 week after surgery. The epithelium has already regenerated, but in disorderly fashion with thick, multilayered parts next to thin parts with cubic cells. Enormous thickening of the subepithelial region demonstrates a large quantity of blood vessels and a loose netting of fibrous tissue. Even the cartilage demonstrates important deformations, of which inconsistent thickness is the most conspicuous. The lumen has narrowed, sometimes to a degree incompatible with life. Twenty weeks after intervention, the oval cricoid ring is transformed into a deformed, sometimes pearshaped ring with sideward or anterior collapse; the lumen has been decreased

*Corresponding author. 
significantly by this deformation [2]. Regardless the depth of the endolaryngeal lesion, which can be superficial or deep, ectopic cartilage is more or less present in all specimens, distributed at random and mostly fibrocartilaginous as revealed by birefringent light. Histologic sections of several larynx specimens of children diagnosed as hamartoma appeared to give similar images with an identical structure [3].

The elastic system, assembled in a combination of oblique running fibres, forms a mantle of concentric layers which are important for the cohesion and simultaneously, the mobility of the different cartilaginous parts of the larynx. It was observed that damage to the endolaryngeal tissues leads to regeneration of the epithelial, mostly respiratory cells, but to a permanent loss of the elastic system and the glands.

The same changes could be shown in the adult operated animals. Scarring and loss of the elastic mantle are the results; the only difference is the preservation of the form of the cartilaginous ring. One explanation of the difference between the young and adult animals can be found in the fact that the cartilage of the cricoid in the adult is much more differentiated, is less flexible and contains more calcium in the intercellular matrix; in short, the cartilage has lost its property of interlocked stresses (Gibson, 1958 and Fry, 1967).

A second factor was revealed by studying the cricoid ring under normal conditions with double refracting light. The perichondrial layer on the outside appears thicker than on the inside. Traumatization of the internal perichondrium not only releases interlocked stresses, which in the young cartilage is not counteracted by the intrisic rigidity of the cartilaginous ring; but also creates a dominance of the pulling forces of the perichondrial fibres on the outside, followed by collapse of the ring during growth.

Interruption ( $=$ splitting) of the cricoid cartilage causes comparable changes in both young and adult specimens. Bilateral splits in the young animal cause the anterior smaller part to stretch considerably as it continues to grow. The larger posterior part opens up and attains a U-like configuration [4]. The same phenomenon of stretching is found in the anteroposterior cricoid split 20 weeks after surgery. In all series of splits at a young age, these changes resulted in an increase of the airway cross sectional area. A reason for this observation was thought to be the tilting action of the cricopharyngeal muscle upon the posterior part, moving the anterior ends of the cricoid in lateral direction [5].

In the adult experimental animal (anterior, bilateral split) the U-form also can be observed, although the anterior part keeps its curved aspect and cannot grow anymore. It hangs loose between the cut ends of the posterior cricoid half. An endolaryngeal lesion combined with a bilateral + anterior cricoid split produced exactly what could be expected after all the above-mentioned results. An extreme inward curving of the grown anterior part goes together with the U-like form, leading to a round airway lumen, smaller in size than normal [6].

To summarize the various factors which can determine the development of a more or less severe subglottic stenosis, the most important features are: 
- regeneration of respiratory epithelium

- thickening / scarring of the soft tissue lining

- loss of elastic fibres-formation of ectopic cartilage

- loss of inner perichondrium leading to more severe deformation of cartilaginous ring

- release of interlocked stresses and muscle action affecting the form of the cricoid

- relationship between wound healing and age

\section{References}

[1] Adriaansen, F.C.P.M., Verwoerd-Verhoef, H.L., v.d. Heul, R.O. and Verwoerd, C.D.A. (1986) A histologic study of the growth of the subglottis after endolaryngeal trauma. Int. J. Ped. Otorhinolaryngol. 12, 205-215.

[2] Adriaansen, F.C.P.M., Verwoerd-Verhoef, H.L., v.d. Heul, R.O. and Verwoerd. C.D.A. (1988) Differential effects of endolaryngeal trauma upon the growth of the subglottis. Int. J. Ped. Otorhinolaryngol. 15, 163-171.

[3] Adriaansen, F.C.P.M., Hoeve, L.J., Verwoerd-Verhoef, H.L., v.d. Heul, R.O. and Verwoerd. C.D.A. (1992) Ectopic cartilage in subglottic stenosis: hamartoma or reaction to trauma? Int. J. Ped. Otorhinolaryngol. 23, 221-227.

[4] Bean, J.K., Verwoerd-Verhoef, H.L., Adriaansen, F.C.P.M. and Verwoerd. C.D.A. (1993) The influence of different types of splits upon the growing cricoid. In: Fior, R. and Pestalozza, G. (Eds.). The Child and the Environment, Elsevier Science Publ., Amsterdam.

[5] Bean, J.K., Verwoerd-Verhoef, H.L. and Verwoerd, C.D.A.. (1994) Intrinsic and extrinsic factors relevant to the morphology of the growing cricoid ring after a combined anterior and posterior cricoid split. Int. J. Ped. Otorhinolaryngol. 29, 129-137.

[6] Verwoerd, C.D.A., Bean, J.K., Adriaansen, F.C.P.M. and Verwoerd-Verhoef, H.L. (1991) Trauma of the cricoid and interlocked stress. Acta Otolaryngol. (Stockh) 111, 403-409. 